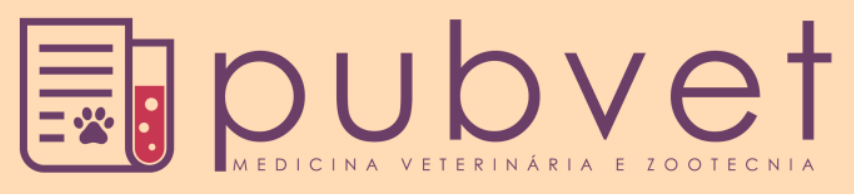

HTTP://DX.DOI.ORG/10.22256/PUBVET.V11N10.1057-1073

\title{
Método matricial de formulação de rações para vacas leiteiras
}

\author{
Rodrigo da Silva Lima ${ }^{1 *}$, José Almir Ferreira Gomes ${ }^{1}$, Edmilson Gomes da Silva ${ }^{1}$, Rafael \\ Santos de Aquino ${ }^{1}$, Francisco Dirceu Duarte Arraes ${ }^{2}$
}

${ }^{1}$ Professores de Zootecnia do Instituto Federal de Educação, Ciência e Tecnologia do Sertão Pernambucano, Salgueiro-PE, Brasil.

${ }^{2}$ Professor de Irrigação e Drenagem do Instituto Federal de Educação, Ciência e Tecnologia do Sertão Pernambucano, Salgueiro-PE, Brasil.

*E-mail para contato: rodrigo.lima@ifsertao-pe.edu.br

\begin{abstract}
RESUMO. Objetivou-se com esse trabalho divulgar a utilização de operações com matrizes na formulação de rações para vacas leiteiras, tendo em vista o grande potencial que essa metodologia apresenta para solucionar parâmetros e incógnitas pré definidas em sistemas de equações lineares. Com a utilização de matrizes é possível solucionar sistemas de equações lineares complexos que seriam de difícil solução por outros meios matemáticos. Dessa forma, utilizando a teoria de matrizes, dependendo do número de parâmetros e incógnitas, podem ser utilizados o cálculo dos determinantes, o método da eliminação de Gauss e o escalonamento de Gauss-Jordan como ferramentas matemáticas para solução dos sistemas. Esses métodos, além de encontrar o valor das variáveis, também permitem fixar ingredientes de forma que se obtenham, na fórmula final, alimentos em uma porcentagem pré-definida, garantindo assim o atendimento das exigências nutricionais requerida pela categoria animal.
\end{abstract}

Palavras chave: nutrição, matrizes, produção animal

\section{Matrix method of formulating rations for dairy cows}

ABSTRACT. The objective of this work was to disclose the use of operations with matrices in the formulation of rations for dairy cows, considering the great potential that this methodology presents to solve pre-defined parameters and unknowns in systems of linear equations. With the use of matrices it is possible to solve systems of complex linear equations that would be difficult to solve by other mathematical means. Thus, using the matrix theory, depending on the number of parameters and unknowns, the determinants calculation, the Gauss elimination method and the Gauss-Jordan scaling can be used as mathematical tools for solving the systems. These methods, besides finding the value of the variables, also allow fixing ingredients in order to obtain in the final formula foods in a pre-defined percentage, thus guaranteeing the fulfilment of the nutritional requirements required by the animal category.

Key words: Nutrition, matrices, animal production

\section{Método matricial de formulación de raciones para vacas lecheras}

RESUMEN. Se objetivó con ese trabajo divulgar la utilización de operaciones con matrices en la formulación de raciones para vacas lecheras, teniendo en vista el gran potencial que esa metodología presenta para solucionar parámetros e incógnitas predefinidas en sistemas de ecuaciones lineales. Con la utilización de matrices es posible solucionar sistemas de ecuaciones lineales complejas que serían de difícil solución por otros medios matemáticos. De esta forma, utilizando la teoría de matrices, dependiendo del número de parámetros e incógnitas, se pueden utilizar el cálculo de los determinantes, el método de la eliminación de Gauss y el escalonamiento de Gauss-Jordan como 
herramientas matemáticas para la solución de los sistemas. Estos métodos, además de encontrar el valor de las variables, también permiten fijar ingredientes de forma que se obtenga, en la fórmula final, alimentos en un porcentaje predefinido, garantizando así la atención de las exigencias nutricionales requerida por la categoría animal.

Palabras clave: nutrición, matrices, producción animal

\section{Introdução}

A nutrição é um dos aspectos mais importantes em um sistema de produção animal. Dietas devidamente balanceadas são necessárias para fornecer o aporte de nutrientes necessários para possibilitar a máxima expressão genética em termos produtivos em qualquer categoria animal. A bovinocultura leiteira de alta produção se destaca pela exigência de elevadas quantidades de nutrientes necessários para a produção de leite. Em alguns casos, quando se somam as diferentes exigências (manutenção, produção, crescimento e gestação) torna-se difícil balancear uma dieta que contemple principalmente o nível energético requerido para atender às necessidades. Assim, o nutricionista deve formular as dietas de forma a oferecer o máximo possível dos nutrientes requeridos pelos animais.

Existem vários métodos de formulação de dietas, sendo os mais utilizados o quadrado de Pearson e os sistemas de equações lineares (também chamado de método algébrico). O quadrado de Pearson se destaca pela facilidade matemática na resolução dos cálculos. Porém, possui a limitação de balancear as dietas apenas para um nutriente, a não ser que se trabalhe com pré-misturas, aumentando significativamente a quantidade de operações matemáticas. Já o método dos sistemas de equações lineares apresenta a possibilidade de balancear vários nutrientes por vez e com vários ingredientes na dieta. Contudo, quando se balanceia uma dieta para vários nutrientes, sendo necessária a resolução de várias equações lineares, o número de operações matemáticas também aumenta significativamente, aumentando a dificuldade e a possibilidade de erro nas operações matemáticas. Um método matemático potencial de formulação de rações, porém muito pouco utilizado, é o método matricial, tendo as operações com matrizes o meio matemático necessário para garantir a resolução das equações que garantem a formulação de dietas com os níveis nutricionais necessários para uma completa nutrição. O método matricial, dependendo do nível de exigência das dietas a serem balanceadas, pode ser matematicamente simples ou bastante complexo. O objetivo desse trabalho é mostrar algumas metodologias matriciais de formulação de dietas para vacas leiteiras, oferecendo mais uma ferramenta para os nutricionistas formuladores desenvolverem suas funções nos sistemas de produção animal.

\section{Exigência nutricional de vacas leiteiras}

As vacas leiteiras, principalmente se forem de alta produção, possuem uma elevada exigência nutricional oriunda do alto custo fisiológico ocasionado principalmente pela lactação. $\mathrm{O}$ cálculo da exigência nutricional deve ser feito de acordo com as condições fisiológicas que o animal se encontra, o que obriga o produtor a separar seu rebanho em lotes o mais uniforme possível.

A formação de lotes ajuda a evitar a superalimentação e a subalimentação de animais com exigência abaixo ou acima, respectivamente, dos níveis nutricionais fornecidos nas dietas. Como exemplo de cálculo de exigência nutricional de vacas leiteiras, vamos utilizar uma vaca com $550 \mathrm{~kg}$ de peso vivo, terceira ordem de lactação e média diária de produção de leite de $25 \mathrm{~kg}$ com $3,5 \%$ de gordura. Vamos seguir as recomendações nutricionais do $\underline{\operatorname{NRC}(2001)}$, segundo tabela 1.

Tabela 1. Fracionamento da exigência nutricional de uma vaca leiteira com $550 \mathrm{~kg}$ de peso vivo, produzindo $25 \mathrm{~kg}$ de leite por dia com $3,5 \%$ de gordura.

\begin{tabular}{lcccccc}
\hline Exigências & $\mathrm{MS}(\mathrm{kg})$ & $\mathrm{NDT}(\mathrm{kg})$ & $\mathrm{PB}(\mathrm{kg})$ & $\mathrm{Ca}(\mathrm{g})$ & $\mathrm{P}(\mathrm{g})$ & $\mathrm{Na}(\mathrm{g})$ \\
\hline Manutenção & 16,5 & 4,37 & 0,386 & 22 & 16 & 2,75 \\
Exigência/kg de leite & & 0,301 & 0,084 & 2,97 & 1,83 & 0,63 \\
Exigência/25 kg de leite & & 7,525 & 2,1 & 74,25 & 45,75 & 15,75 \\
Exigência total & 16,5 & 11,89 & 2,486 & 96,25 & 61,75 & 18,50 \\
\hline
\end{tabular}

Fonte: Adaptado de NRC (1989). *Exigência recomendada pelo NRC (2001). 
Verifica-se que ao ser convertida a exigência para valores percentuais, obtemos uma exigência de $72,06 \%$ de NDT; $15,06 \%$ de PB; $0,58 \%$ de cálcio, $0,37 \%$ de fósforo e $0,11 \%$ de sódio. Ressalta-se que essa exigência em termos quantitativos ( $\mathrm{kg}$ ou $\mathrm{g}$ ) ou percentuais devem ser balanceados para uma capacidade de consumo equivalente a $16,5 \mathrm{~kg}$ de matéria seca por dia. Após o cálculo da exigência nutricional de uma determinada categoria animal, o nutricionista deve avaliar e escolher os alimentos disponíveis para utilizar na formulação da dieta a ser balanceada.

A escolha dos alimentos deve ser feita segundo sua disponibilidade na propriedade, a qualidade nutricional dos alimentos, o custo de cada alimento e de acordo com os objetivos produtivos a serem alcançados. A escolha correta de cada alimento é uma decisão importante no planejamento nutricional do rebanho. Geralmente, existem várias possibilidades de alimentos volumosos e concentrados que podem compor as dietas animais, sendo alguns deles mostrados na tabela 2. Após calcular a exigência nutricional e escolher os alimentos que vão compor as dietas, o nutricionista deve utilizar o método matemático de formulação que possibilite o mais completo atendimento dos níveis nutricionais requeridos.

Existem muitos softwares de formulação de ração gratuitos e privados disponíveis no mercado que podem ser utilizados na formulação das dietas. Contudo, a formulação manual ainda é uma possibilidade que não pode ser desconsiderada pelos formuladores, tendo em vista que os softwares apenas fazem as operações matemáticas seguindo os critérios pré-estabelecidos pelo nutricionista. A avaliação da fórmula obtida e a qualidade e praticidade das dietas são uma prática necessária dos nutricionistas, podendo influenciar diretamente o sucesso do empreendimento.

Tabela 2. Composição química de alimentos utilizados em dietas para vacas leiteiras (valores percentuais com base na matéria seca).

\begin{tabular}{|c|c|c|c|c|c|c|c|}
\hline Alimentos & MS & NDT & $\mathrm{PB}$ & PDR & $\mathrm{Ca}$ & $\mathrm{P}$ & $\mathrm{Na}$ \\
\hline Silagem de milho & 30,92 & 64,27 & 7,26 & 5,35 & 0,30 & 0,19 & 0,03 \\
\hline Silagem de sorgo & 30,82 & 57,23 & 6,69 & 2,14 & 0,30 & 0,18 & 0,02 \\
\hline Silagem de capim elefante & 26,81 & 58,08 & 5,84 & $2,92 *$ & 0,35 & 0,13 & 0,01 \\
\hline Feno de tifton 85 & 88,96 & 55,62 & 8,96 & 3,93 & 0,42 & 0,17 & 0,02 \\
\hline Milho moído & 87,64 & 87,24 & 9,11 & 3,75 & 0,03 & 0,25 & 0,02 \\
\hline Farelo de trigo & 88,01 & 72,43 & 16,63 & 11,97 & 0,22 & 1,00 & 0,04 \\
\hline Farelo de algodão & 89,95 & 68,31 & 40,90 & 22,08 & 0,24 & 1,00 & 0,04 \\
\hline Farelo de soja & 88,61 & 81,54 & 48,78 & 31,88 & 0,34 & 0,58 & 0,02 \\
\hline Calcário calcítico & 99,27 & - & - & - & 37,33 & - & - \\
\hline Fosfato bicálcico & 98,57 & - & - & - & 24 & 18,48 & - \\
\hline Melaço de cana de açúcar & 73,98 & 70,0 & 3,66 & $1,83 *$ & - & - & - \\
\hline Sal comum & 99,0 & - & - & - & - & - & 39,7 \\
\hline Ureia & - & - & 281,25 & 281,25 & - & - & - \\
\hline
\end{tabular}

O método matricial de formulação de rações se baseia especificamente em encontrar uma solução matemática para equações lineares através de operações com matrizes. Assim, antes de criar as matrizes que serão manipuladas pelo formulador, é necessário criar as equações (com coeficientes e incógnitas) que atendam às necessidades nutricionais quando forem solucionadas. A título de exemplo, serão utilizadas as exigências nutricionais determinadas na Tabela 1 .

\section{Elaboração das equações lineares}

As equações que posteriormente serão convertidas em matrizes devem ser elaboradas para atender as necessidades de consumo e de cada nutriente. Com isso, deve-se elaborar uma equação para atender as exigências de matéria seca (MS), nutrientes digestíveis totais (NDT), proteína bruta $(\mathrm{PB})$, proteína degradada no rúmen (PDR), cálcio $(\mathrm{Ca})$, fósforo $(\mathrm{P})$ etc. Ressalta-se que quanto mais equações forem utilizadas nos cálculos, maior será o tamanho da matriz e também a quantidade de operações matemáticas necessárias para solucioná-las. Assim, propõe-se a elaboração de equações apenas para MS, NDT, PB, PDR, Ca, $\mathrm{P}$ e $\mathrm{Na}$, deixando uma pequena margem para inclusão de suplementos que possam satisfazer as necessidades dos outros minerais e vitaminas na ração ou dieta. 
As equações devem ser compostas com coeficientes e incógnitas. Os coeficientes devem ser os teores de MS ou nutrientes que o alimento (incógnita) apresenta em sua composição química, e igualada ao valor da exigência de MS ou nutriente calculado. Ressalta-se que os alimentos que irão compor uma equação para balancear um nutriente deverão possuir teores (porcentagem) desse respectivo nutriente acima e abaixo do teor que o nutriente deverá apresentar na ração ou dieta calculada. Por exemplo, se precisamos fazer uma fórmula que apresente $70 \%$ de NDT, essa fórmula só será possível se houver em sua composição alimentos que tenham menos que os $70 \%$ de NDT requeridos e alimentos que possuam mais de $70 \%$ de NDT. Ainda como exemplo, para montar uma equação para proporcionar na ração final $70 \%$ de NDT poderíamos utilizar feno de tífton 85 que possui 55\% (nível a baixo da exigência) de NDT e milho moído que apresenta $87 \%$ (nível a cima da exigência) de NDT.

Utilizando os alimentos Silagem de capim elefante e Farelo de soja para elaborar a equação que atenda a exigência de NDT, a equação ficaria da seguinte forma:

\section{$0,5808 \mathrm{SCE}+0,8164 \mathrm{FS}=11,89$}

Onde:

SCE e FS são as quantidades (em kg) de MS de silagem de capim elefante e farelo de soja a serem encontrados, respectivamente;

0,5808 e 0,8164 são os teores de NDT da silagem de capim elefante e farelo de soja, respectivamente;

11,89 é a quantidade, em $\mathrm{kg}$, de NDT que a vaca necessita consumir diariamente.

Contudo, a resolução matemática dessa equação atenderá apenas as necessidades de consumo de NDT, ficando os outros nutrientes desbalanceados na dieta. Assim, recomenda-se a elaboração de outras equações para balancear conjuntamente vários nutrientes. A título de exemplo, vamos elaborar a equação, com os mesmos alimentos, para balancearmos ao mesmo tempo o NDT e a PB da dieta. A elaboração da equação para a $\mathrm{PB}$ segue o mesmo requisito da equação do NDT, trocando apenas os coeficientes e o valor da igualdade. Ficaria assim:

0,0584 SCE + 0,4878 FS $=2,486$

Onde:
0,0584 e 0,4878 são os teores de PB da silagem de capim elefante e farelo de soja, respectivamente;

SCE e FS são as quantidades (em $\mathrm{kg}$ ) de silagem de capim elefante e farelo de soja, respectivamente, necessários para atender a exigência de $\mathrm{PB}$;

2,486 é a exigência diária, em $\mathrm{kg}$, de PB que a silagem de capim elefante e o farelo de soja devem fornecer.

Perceba que para balancear conjuntamente o NDT e a PB as duas equações deverão apresentar uma mesma solução matemática para as duas variáveis, ou seja, o mesmo valor de silagem de capim elefante e de farelo de soja para as duas equações. Agora, com essas duas equações elaboradas pode-se utilizar o método matemático para resolução das variáveis.

\section{Elaboração das matrizes}

Organizando as equações elaboradas em um sistema, para resolução conjunta, teremos:

$$
0,5808 \mathrm{SCE}+0,8164 \mathrm{FS}=11,89
$$$$
0,0584 \mathrm{SCE}+0,4878 \mathrm{FS}=2,486
$$

Ressalta-se que todo sistema de equações lineares possui uma matriz associada. Assim, a matriz dos coeficientes referente a esse sistema de equações seria:

$\left|\begin{array}{cc}0,5808 & 0,8164 \\ 0,0584 & 0,4878\end{array}\right|$

Observe que essa matriz é composta pelos coeficientes que multiplicam as incógnitas presentes no sistema. Note que essa matriz não contempla os valores a direita da igualdade, ou seja, os valores referentes às exigências de NDT e PB (11,89 e 2,486, respectivamente). Esses valores, na linguagem matemática, podem ser chamados de termos independentes. Matrizes que além dos coeficientes apresentam os valores dos termos independentes são chamadas de matrizes aumentadas. Nesse exemplo, seria:

$\left|\begin{array}{ll:l}0,5808 & 0,8164 & 11,89 \\ 0,0584 & 0,4878 & 2,486\end{array}\right|$

A linha tracejada entre os valores dos coeficientes e os valores dos termos independentes serve para visualizar o que seria a matriz dos 
coeficientes e a matriz aumentada referente ao sistema correspondente. É válido lembrar que matrizes são compostas de linhas (horizontalmente) e colunas (verticalmente), assim, no nosso exemplo da matriz dos coeficientes temos uma matriz de ordem $2 \times 2$ (duas linhas e duas colunas) e no caso da matriz aumentada temos uma matriz de ordem $2 \times 3$ (duas linhas e três colunas). Comparando as linhas e as colunas com o sistema que originou a matriz, verificamos que:

- A primeira linha corresponde a equação elaborada para o NDT;

- A segunda linha corresponde a equação elaborada para a PB;

- A primeira coluna (coluna da esquerda) corresponde a incógnita silagem de capim elefante (SCE);

- A segunda coluna (coluna do meio) corresponde a incógnita farelo de soja (FS);

- A terceira coluna (coluna da direita) corresponde aos valores dos termos independentes, para os quais as equações devem ser solucionadas.

Com o conhecimento detalhado dos componentes da matriz é possível utilizar os meios matemáticos para solucionar as incógnitas. Existem vários métodos matriciais que podem ser utilizados.

\section{Método dos determinantes}

Utilizando a matriz elaborada anteriormente, vamos utilizar operações com determinantes para encontrarmos os valores de silagem de capim elefante e farelo de soja que satisfaçam o sistema de equações que deu origem a matriz. Inicialmente vamos calcular o determinante da matriz dos coeficientes, sem a coluna dos termos independentes. Para calcular o determinante em matrizes $2 \times 2$, basta apenas subtrair o produto da diagonal principal do produto da diagonal secundária. $\mathrm{O}$ cálculo do determinante ficaria da seguinte forma:

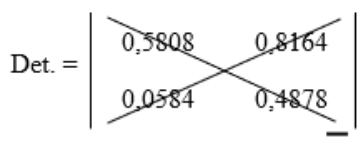

Det. $=(0,5808 \times 0,4878)-(0,8164 \times 0,0584)$

Det. $=0,2356$
Agora, sabendo que a primeira coluna da matriz é referente a incógnita da silagem de capim elefante (SCE), vamos modificar a matriz dos coeficientes substituindo a primeira coluna (referente a silagem de capim elefante) pela coluna dos termos independentes (terceira coluna da matriz aumentada). Essa matriz ficaria da seguinte forma:

$\left|\begin{array}{ll}11,89 & 0,8164 \\ 2,486 & 0,4878\end{array}\right|$

Agora, vamos calcular o determinante dessa matriz e vamos chamá-lo de Det.SCE:

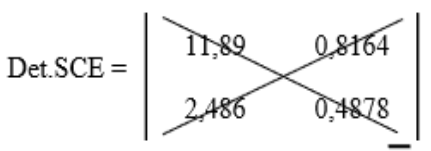

Det.SCE $=(11,89 \times$ X 0,4878$)-(0,8164 \times 2,486)$

Det.SCE $=3,7703$

Agora, vamos substituir a segunda coluna (referente ao farelo de soja) pela coluna dos termos independentes e calcular o determinante. A primeira coluna, referente a silagem de capim elefante, permanece na matriz.

$\left|\begin{array}{cc}0,5808 & 11,89 \\ 0,0584 & 2,486\end{array}\right|$

Da mesma forma, vamos calcular o determinante dessa matriz e vamos chamá-lo de Det.FS:

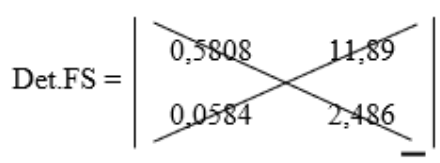

Det.FS $=(0,5808 \times 2,486)-(11,89 \times 0,0584)$

Det.FS $=0,7494$

Para encontrar o valor de SCE que atenda aos requisitos matemáticos no sistema de equações, é necessário apenas dividir o Det.SCE pelo Det., obtendo o seguinte valor:

$\mathrm{SCE}=$ Det.SCE $/$ Det $=3,7703 / 0,2356$

$\mathrm{SCE}=16,0 \mathrm{~kg}$ de MS 
Da mesma forma, para encontrar o valor de FS que atenda aos requisitos matemáticos no sistema de equações, é necessário dividir o Det.FS pelo Det., obtendo o seguinte resultado:

$\mathrm{FS}=$ Det.FS $/$ Det. $=0,7494 / 0,2356$

$\mathrm{FS}=3,18 \mathrm{~kg}$ de $\mathrm{MS}$

Assim, com a manipulação matricial do sistema de equações verificamos que é necessário fornecer $16,0 \mathrm{~kg}$ de MS de silagem de capim elefante e $3,18 \mathrm{~kg}$ de MS de farelo de soja para que as exigências de NDT e PB sejam atendidas. No entanto, ao avaliar a capacidade de consumo do animal utilizado nesse exemplo, observamos que sua capacidade média de consumo é de $16,5 \mathrm{~kg}$ de MS por dia. Desta forma, a quantidade somada de silagem de capim elefante e farelo de soja $(19,18$ kg de MS) necessária para consumo diário está acima da capacidade de consumo pelo animal. Essa situação implicaria em um consumo menor que $\mathrm{o}$ necessário e $\mathrm{o}$ não atendimento das exigências nutricionais, implicando em diminuição da produção e demais problemas que podem ser causados por subnutrição. Verifica-se então que é necessário a formulação de dietas que forneçam os nutrientes necessários dentro da capacidade de consumo do animal. Os métodos matriciais de formulação de rações possibilitam o atendimento desses requisitos. Para demonstrar, vamos utilizar o exemplo anterior, só que levando em consideração os $16,5 \mathrm{~kg}$ de MS possíveis de serem consumidos pela vaca utilizada como exemplo.

No exemplo anterior foram elaboradas as equações referentes ao consumo necessário de NDT e PB. Para que seja possível formular uma dieta que atenda as exigências nutricionais com um consumo limitado, também deve ser elaborada uma equação que limite o consumo de matéria seca segundo a capacidade do animal. Para o exemplo anterior, a equação ficaria da seguinte forma:

$$
\mathrm{SCE}+\mathrm{FS}=16,5
$$

Desta forma, ao inserir essa equação no sistema composto pelas equações do NDT e PB teríamos um sistema com três equações e duas variáveis. Ao converter esse sistema em uma matriz, teríamos uma matriz de ordem $3 \times 2$ (três linhas e duas colunas), no caso da matriz dos coeficientes. Contudo, para facilitar os métodos matemáticos de resolução das incógnitas, é necessário transformar essa matriz 3 X 2 em uma matriz 3 X 3, ou seja, uma matriz quadrada. Para isso, é necessário apenas incluir mais um alimento para ser utilizado na formulação, aumentando mais uma incógnita, conferindo mais uma coluna a matriz. Como exemplo vamos acrescentar o milho moído (MM) nas equações, deixando-as da seguinte forma:

$$
\begin{aligned}
& \mathrm{MM}+\mathrm{SCE}+\mathrm{FS}=16,5 \\
& 0,8724 \mathrm{MM}+0,5808 \mathrm{SCE}+0,8164 \mathrm{FS}=11,89 \\
& 0,0911 \mathrm{MM}+0,0584 \mathrm{SCE}+0,4878 \mathrm{FS}=2,486
\end{aligned}
$$

Agora vamos converter esse sistema na matriz dos coeficientes:

$\left|\begin{array}{ccc}1 & 1 & 1 \\ 0,8724 & 0,5808 & 0,8164 \\ 0,0911 & 0,0584 & 0,4878\end{array}\right|$

A matriz aumentada ficaria da seguinte forma:

$\left|\begin{array}{ccc:c}1 & 1 & 1 & 16,5 \\ 0,8724 & 0,5808 & 0,8164 & 11,89 \\ 0,0911 & 0,0584 & 0,4878 & 2,486\end{array}\right|$

A utilização do cálculo dos determinantes em matrizes $3 \times 3$ para resolução das incógnitas também é possível. Contudo, em matrizes 3 X 3 requer uma metodologia diferente para encontrar os determinantes. O método para calcular o determinante que será utilizado nesse exemplo será o método de Sarrus. Esse método consiste em repetir as duas primeiras colunas ao lado das três colunas. Veja como ficaria:

$\left|\begin{array}{ccc:cc}1 & 1 & 1 & 1 & 1 \\ 0,8724 & 0,5808 & 0,8164 & 0,8724 & 0,5808 \\ 0,0911 & 0,0584 & 0,4878 & 0,0911 & 0,0584\end{array}\right|$

Após a matriz montada, deve-se somar os produtos das diagonais principais e subtrair dos produtos das diagonais secundárias. Seria da seguinte forma:

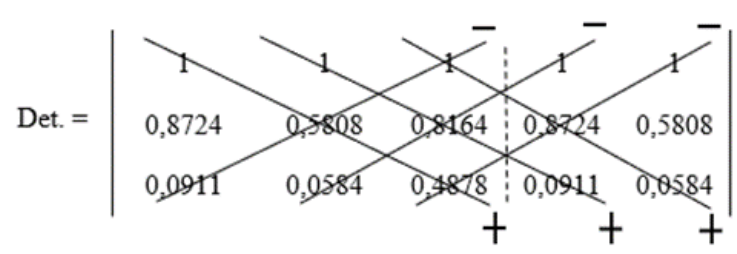




$$
\begin{aligned}
\text { Det. }= & (1 \times 0,5808 \times 0,4878)+(1 \times 0,8164 \times \\
& 0,0911)+(1 \times 0,8724 \times 0,0584)-(1 \times \\
& 0,8724 \times 0,4878)-(1 \times 0,8164 \times 0,0584) \\
& -(1 \times 0,5808 \times 0,0911)
\end{aligned}
$$

Det. $=-0,1175$

Da mesma forma do exemplo anterior, após o cálculo do determinante da matriz dos coeficientes, é necessário fazer o cálculo dos determinantes das matrizes com a substituição das colunas das incógnitas pela coluna dos termos independentes. A matriz da incógnita milho moído ficaria da seguinte forma:

$\left|\begin{array}{ccc}16,5 & 1 & 1 \\ 11,89 & 0,5808 & 0,8164 \\ 2,486 & 0,0584 & 0,4878\end{array}\right|$

Agora vamos calcular o determinante dessa matriz utilizando a regra de Sarrus conforme exemplo anterior:

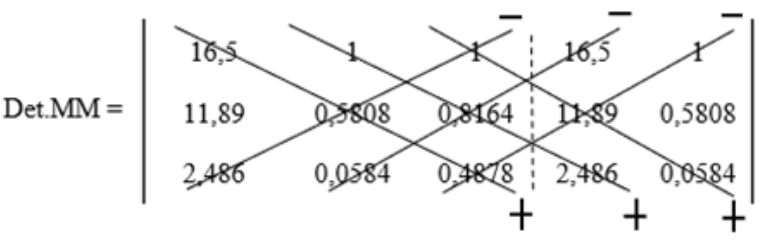

Det.MM $=(16,5 \times 0,5808 \times 0,4878)+(1 \times 0,8164$ x 2,486$)+(1 \times 11,89 \times 0,0584)-(1 \times$ $11,89 \times 0,4878)-(16,5 \times 0,8164 \times$ $0,0584)-(1 \times 0,5808 \times 2,486)$

Det.MM $=-0,6318$

Agora vamos elaborar a matriz substituindo a coluna da incógnita SCE pelos termos independentes e calcular o determinante:

$\left|\begin{array}{ccc}1 & 16,5 & 1 \\ 0,8724 & 11,89 & 0,8164 \\ 0,0911 & 2,486 & 0,4878\end{array}\right|$

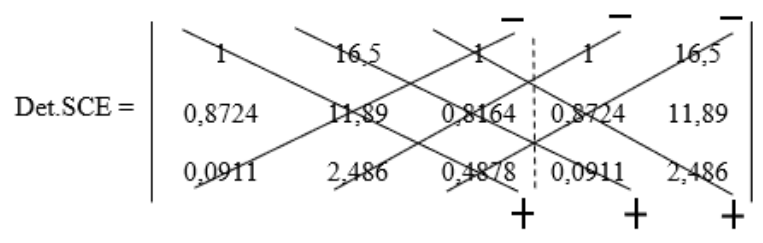

Det.SCE $=(1 \times 11,89 \times 0,4878)+(16,5 \times 0,8164$ x 0,0911$)+(1 \times 0,8724 \times 2,486)-(16,5 \times$ $0,8724 \times 0,4878)-(1 \times 0,8164 \times 2,486)-$
$(1 \times 11,89 \times 0,0911)$

Det.SCE $=-0,9385$

Da mesma forma, vamos elaborar a matriz substituindo a coluna da incógnita FS pelos termos independentes e calcular o determinante:

$\left|\begin{array}{ccc}1 & 1 & 16,5 \\ 0,8724 & 0,5808 & 11,89 \\ 0,0911 & 0,0584 & 2,486\end{array}\right|$

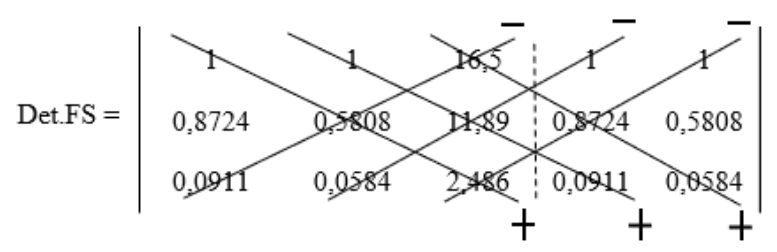

Det.FS $=(1 \times 0,5808 \times 2,486)+(1 \times 11,89 \times$ $0,0911)+(16,5 \times 0,8724 \times 0,0584)-(1 \times$ $0,8724 \times 2,486)-(1 \times 11,89 \times 0,0584)-$ $(16,5 \times 0,5808 \times 0,0911)$

Det.FS $=-0,3684$

Agora, vamos dividir os determinantes das incógnitas pelo determinante da matriz dos coeficientes e encontrar os valores de milho moído (MM), silagem de capim elefante (SCE) e farelo de soja (FS):

$$
\begin{aligned}
& \mathrm{MM}=\text { Det.MM } / \text { Det. }=-0,6318 /-0,1175 \\
& \mathrm{MM}=5,38 \mathrm{~kg} \text { de MS } \\
& \mathrm{SCE}=\text { Det.SCE } / \text { Det. }=-0,9385 /-0,1175
\end{aligned}
$$

$\mathrm{SCE}=7,99 \mathrm{~kg}$ de MS

FS $=$ Det.FS $/$ Det. $=-0,3684 /-0,1175$

$\mathrm{FS}=3,13 \mathrm{~kg}$ de $\mathrm{MS}$

Então verifica-se que a quantidade de MS de milho moído, silagem de capim elefante e farelo de soja que atendem as exigências nutricionais são 5,38; 7,99 e 3,13 kg, respectivamente. Observe que o somatório das quantidades desses três alimentos equivale exatamente aos $16,5 \mathrm{~kg}$ de MS que a vaca utilizada no exemplo pode consumir. Note também que existe uma razão volumoso:concentrado igual a 48:52, conferindo uma boa fonte de fibras nessa dieta, principalmente devido a silagem de capim elefante apresentar, geralmente, uma boa quantidade de fibra fisicamente efetiva necessária para um perfeito funcionamento ruminal. Desta forma, as 
necessidades de energia, proteína e fibras devem estar satisfeitas.

\section{Método da Eliminação de Gauss}

Como já mostrado, o exemplo anterior foi elaborado para balancear apenas o NDT e a PB dentro de um consumo potencial de $16,5 \mathrm{~kg}$ de MS. Não houve balanceamento de cálcio, fósforo e outros nutrientes muito importantes principalmente na dieta de vacas leiteiras. Contudo, também é possível fazer o cálculo da dieta, por meios matriciais, de forma que esses minerais também estejam balanceados com a solução das incógnitas. Vamos agora ampliar a qualidade da dieta balanceada, ainda utilizando a mesma vaca como exemplo, acrescentando agora no sistema as equações para balanceamento do cálcio e do fósforo, resultando em cinco equações lineares. Assim, como já mencionado anteriormente, para facilitar as operações matriciais, devemos elaborar uma matriz associada ao sistema em questão de forma que apresente o mesmo número de linhas e colunas (matriz quadrada). Para isso, devemos acrescentar nas equações mais dois ingredientes, um fonte de cálcio, enquanto que o outro seja fonte de fósforo. Nesse exemplo vamos utilizar o calcário calcítico (como fonte de cálcio) e o fosfato bicálcico (como fonte de fósforo). Definidos esses critérios, o novo sistema de equações ficará da seguinte forma:

$$
\begin{aligned}
& \mathrm{CC}+\mathrm{FB}+\mathrm{MM}+\mathrm{SCE}+\mathrm{FS}=16,5 \\
& 0 \mathrm{CC}+0 \mathrm{FB}+0,8724 \mathrm{MM}+0,5808 \mathrm{SCE}+0,8164 \\
& \mathrm{FS}=11,89 \\
& 0 \mathrm{CC}+0 \mathrm{FB}+0,0911 \mathrm{MM}+0,0584 \mathrm{SCE}+0,4878 \\
& \mathrm{FS}=2,486 \\
& 0,3733 \mathrm{CC}+0,24 \mathrm{FB}+0,0003 \mathrm{MM}+0,0035 \mathrm{SCE} \\
& +0,0034 \mathrm{FS}=0,09625 \\
& 0 \mathrm{CC}+0,1848 \mathrm{FB}+0,0025 \mathrm{MM}+0,0013 \mathrm{SCE}+ \\
& 0,0058 \mathrm{FS}=0,06175
\end{aligned}
$$

Perceba que as três primeiras equações são as mesmas do exemplo anterior, apenas acrescidas do calcário calcítico (CC) e do fosfato bilcálcico (FB). Note que os coeficientes do $\mathrm{CC}$ e do FB nas equações do NDT e da PB são iguais a zero, já que esses ingredientes não fornecem energia e proteína para os animais. Adicionalmente, foram elaboradas a quarta equação, referente as necessidades nutricionais de cálcio, e a quinta equação, referente as necessidades nutricionais de fósforo. Os coeficientes da quarta e quinta equação são os respectivos teores de cálcio e fósforo que os ingredientes apresentam. Note que o sistema se tornou muito maior e mais complexo, sendo difícil a solução das incógnitas manipulando matematicamente esse sistema de equações. Contudo, existem meios matriciais que permitem a solução das incógnitas com operações matemáticas mais simples. Para isso, vamos elaborar a matriz aumentada associada a esse sistema, ficando da seguinte forma:

$\left|\begin{array}{ccccc:c}1 & 1 & 1 & 1 & 1 & 16,5 \\ 0 & 0 & 0,8724 & 0,5808 & 0,8164 & 11,89 \\ 0 & 0 & 0,0911 & 0,0584 & 0,4878 & 2,486 \\ 0,3733 & 0,24 & 0,0003 & 0,0035 & 0,0034 & 0,09625 \\ 0 & 0,1848 & 0,0025 & 0,0013 & 0,0058 & 0,06175\end{array}\right|$

Contrariamente ao exemplo anterior, não utilizaremos cálculos de determinantes para encontrarmos a solução das incógnitas devido à dificuldade matemática para calcular os determinantes de uma matriz 5 x 5 . Nesse exemplo, vamos utilizar uma metodologia chamada Eliminação de Gauss, que irá simplificar essa matriz a um ponto que será possível a solução das variáveis. Esse método consiste em zerar, através de operações matemáticas, todos os valores abaixo da diagonal principal, possibilitando a conversão da matriz escalonada em um sistema de equações simples e de fácil solução. Essas operações matemáticas consistem na multiplicação dos valores de uma linha por um valor não nulo, com possível adição e subtração dos produtos desses valores. Também é possível haver a troca de linhas da matriz. Devemos iniciar com os valores que estão abaixo do primeiro elemento da diagonal principal. Quando todos os valores abaixo de um elemento da diagonal principal estiverem zerados, passa-se para o elemento a direita na diagonal principal, utilizando as operações matemáticas para zerar os valores abaixo dele e assim sucessivamente. $\mathrm{O}$ método da eliminação de Gauss nessa matriz $5 \mathrm{X}$ 5 seria da seguinte forma:

\begin{tabular}{|ccccc:c}
11 & 1 & 1 & 1 & 1 & 16,5 \\
0 & 0 & 0,8724 & 0,5808 & 0,8164 & 11,89 \\
0 & 0 & 0,0911 & 0,0584 & 0,4878 & 2,486 \\
0,3733 & 0,24 & 0,0003 & 0,0035 & 0,0034 & 0,09625 \\
0 & 0,1848 & 0,0025 & 0,0013 & 0,0058 & 0,06175
\end{tabular} \mid


O primeiro elemento da diagonal principal está circulado. Note que é o elemento que está na posição linha 1, coluna 1. Perceba que apenas o elemento da linha 4, coluna 1 não está zerado. Para zerá-lo devemos fazer a seguinte operação: L4 <= L4 - 0,3733 L1 (para formar a nova linha 4, vamos subtrair os valores da linha 4 pelos produtos da multiplicação da constante 0,3733 pelos valores da linha 1). A matriz ficará assim:

$$
\begin{array}{|ccccc:c}
1 & 1 & 1 & 1 & 1 & 16,5 \\
0 & 0 & 0,8724 & 0,5808 & 0,8164 & 11,89 \\
0 & 0 & 0,0911 & 0,0584 & 0,4878 & 2,486 \\
0 & -0,1333 & -0,373 & -0,3698 & -0,3699 & -6,0632 \\
0 & 0,1848 & 0,0025 & 0,0013 & 0,0058 & 0,06175
\end{array} \mid
$$

Observe que todos os elementos da coluna 1 que estão abaixo da diagonal principal já estão zerados. Passa-se para o próximo elemento da diagonal principal (valor a direita que está circulado na linha 2, coluna 2). Contudo, perceba que o valor é zero. Assim, vamos trocar a linha 2 pela linha 5, para evitar que os elementos da diagonal principal possuam o valor zero, impossibilitando a solução das incógnitas. A matriz ficará da seguinte forma:

$$
\begin{array}{|ccccc:c}
1 & 1 & 1 & 1 & 1 & 16,5 \\
0 & 0,1848 & 0,0025 & 0,0013 & 0,0058 & 0,06175 \\
0 & 0 & 0,0911 & 0,0584 & 0,4878 & 2,486 \\
0 & -0,1333 & -0,373 & -0,3698 & -0,3699 & -6,0632 \\
0 & 0 & 0,8724 & 0,5808 & 0,8164 & 11,89
\end{array} \mid
$$

Observe que apenas o elemento da linha 4 abaixo da diagonal principal não está zerado. Assim, vamos fazer a seguinte operação para obter o valor nulo: $\mathrm{L} 4<=\mathrm{L} 4+0,7213 \mathrm{~L} 2$. A constante 0,7213 foi gerada pela divisão o valor $-0,1333$ (valor a ser anulado) pelo valor 0,1848 (valor da diagonal principal). Ressalta-se que a geração dessas constantes sempre ocorre dividindo-se o valor a ser anulado pelo valor da diagonal principal ou vice-versa, sempre dependendo de qual valor é maior ou menor. Perceba que com essas divisões quase sempre serão obtidas frações infinitas. Nos exemplos demonstrados nesse manual, foram utilizadas apenas as primeiras quatro casas decimais, de forma que os cálculos não fiquem exaustivos. A nova matriz ficará da seguinte forma:

$$
\begin{array}{|ccccc:c|}
1 & 1 & 1 & 1 & 1 & 16,5 \\
0 & 0,1848 & 0,0025 & 0,0013 & 0,0058 & 0,06175 \\
0 & 0 & 0,0911 & 0,0584 & 0,4878 & 2,486 \\
0 & 0 & -0,3711 & -0,3688 & -0,3657 & -6,0186 \\
0 & 0 & 0,8724 & 0,5808 & 0,8164 & 11,89
\end{array} \mid
$$

Note que os elementos abaixo da diagonal principal na segunda coluna já estão zerados, passando-se para o próximo elemento da diagonal principal na terceira coluna. Para zerar os elementos abaixo da diagonal principal na terceira coluna vamos fazer as seguintes operações: L5 <= 9,5762 L3 - L5; L4 <= 4,0735 L3 + L4. A nova matriz será:

$\begin{array}{|ccccc:c|}1 & 1 & 1 & 1 & 1 & 16,5 \\ 0 & 0,1848 & 0,0025 & 0,0013 & 0,0058 & 0,06175 \\ 0 & 0 & 0,0911 & 0,0584 & 0,4878 & 2,486 \\ 0 & 0 & 0 & -0,1309 & 1,6213 & 4,1081 \\ 0 & 0 & 0 & -0,0215 & 3,8548 & 11,9164\end{array} \mid$

Desta forma, apenas o elemento abaixo da diagonal principal da quarta coluna e quinta linha não está zerado. Para zerar este valor vamos aplicar a operação: L5 $<=-6,0883$ L5 + L4, deixando a nova matriz da seguinte forma:

\begin{tabular}{|ccccc:c}
1 & 1 & 1 & 1 & 1 & 16,5 \\
0 & 0,1848 & 0,0025 & 0,0013 & 0,0058 & 0,06175 \\
0 & 0 & 0,0911 & 0,0584 & 0,4878 & 2,486 \\
0 & 0 & 0 & $-0,1309$ & 1,6213 & 4,1081 \\
0 & 0 & 0 & 0 & $-21,848$ & $-68,443$
\end{tabular} \mid

Observe que todos os elementos abaixo da diagonal principal estão zerados, tornando a matriz escalonada e com possível solução das incógnitas. Para isso, basta converter essa matriz novamente em um sistema de equações e solucionar cada equação. $\mathrm{O}$ sistema e a solução das equações são:

$$
\begin{array}{r}
\mathrm{CC}+\mathrm{FB}+\mathrm{MM}+\mathrm{SCE}+\mathrm{FS}=16,5 \\
0,1848 \mathrm{FB}+0,0025 \mathrm{MM}+0,0013 \mathrm{SCE}+0,0058 \mathrm{FS} \\
=0,06175 \\
0,0911 \mathrm{MM}+0,0584 \mathrm{SCE}+0,4878 \mathrm{FS}=2,486 \\
-0,1309 \mathrm{SCE}+1,6213 \mathrm{FS}=4,1081 \\
-21,8478 \mathrm{FS}=-68,4425
\end{array}
$$


Resolvendo;

$\mathrm{FS}=-68,4425 /-21,8478$

$\mathrm{FS}=3,132 \mathrm{~kg}$ de $\mathrm{MS}$

- 0,1309 SCE = 4,1081 - $(1,6213$ x 3,132)

$\mathrm{SCE}=7,408 \mathrm{~kg}$ de $\mathrm{MS}$

$0,0911 \mathrm{MM}=2,486-(0,4878 \times 3,132)-(0,0584$ $\mathrm{x} 7,408$ )

$\mathrm{MM}=5,77 \mathrm{~kg}$ de $\mathrm{MS} \quad 0,1848 \mathrm{FB}=0,06175-$ $(0,0058 \times 3,132)-(0,0013 \times 7,408)-$ $(0,0025 \times 5,77)$

$\mathrm{FB}=0,105 \mathrm{~kg}$ de $\mathrm{MS}$

$\mathrm{CC}=16,5-3,132-7,408-5,77-0,105$

$\mathrm{CC}=0,085 \mathrm{~kg}$ de $\mathrm{MS}$

Assim, obtemos a seguinte fórmula para atender as exigências:

- Farelo de soja $=3,132 \mathrm{~kg} \mathrm{MS}$

- Silagem de capim elefante $=7,408 \mathrm{~kg}$ MS

- Milho moído = 5,77 kg MS

- Fosfato bicálcico $=0,105 \mathrm{~kg}$ MS

- Calcário calcítico $=0,085 \mathrm{~kg}$ MS

Total $=16,5 \mathrm{~kg}$ de MS

Com essa fórmula, além do balanceamento da energia, proteína, cálcio e fósforo necessários, verifica-se uma porcentagem de $44,8 \%$ de volumoso e 55,2\% de concentrado. Nessa proporção o volumoso provavelmente fornece a quantidade de fibras fisicamente efetiva suficiente para estimular uma boa motilidade ruminal. Contudo, poderíamos calcular uma dieta ainda mais completa, em termos nutricionais, por meio matricial. Para isso, vamos adicionar no nosso sistema mais uma equação para balancear a proteína degradada no rúmen (PDR), garantindo uma fonte disponível de nitrogênio para utilização pelos microrganismos ruminais. Segundo Valadares Filho (2006), nas condições tropicais brasileiras, cada $\mathrm{kg}$ de NDT ingerido pode produzir $120 \mathrm{~g}$ de proteína microbiana no rúmen, sendo as exigências de PDR equivalente ao produto da quantidade de NDT ingerido pelo valor 120 e multiplicado pelo fator 1,11 (fator de correção). No nosso exemplo, há uma exigência de $11,89 \mathrm{~kg}$ de NDT, apresentando, assim, uma exigência de $1,583 \mathrm{~kg}$ de PDR $(11,89$ x 0,12 x 1,11). Vamos utilizar ureia (U) como principal ingrediente fonte de proteína degradada no rúmen. Vale lembrar que esse ingrediente também deverá ser adicionado nas outras equações do sistema. Também vamos deixar uma margem na capacidade de consumo equivalente a $0,1 \mathrm{~kg}$ para inclusão de suplemento micromineral e vitamínico na dieta para atender a demanda de microminerais e vitaminas. Assim, o novo consumo de matéria seca deverá ser corrigido para $16,4 \mathrm{~kg} / \mathrm{dia}$. O novo sistema de equações ficará assim:

$$
\begin{aligned}
& \mathrm{U}+\mathrm{CC}+\mathrm{FB}+\mathrm{MM}+\mathrm{SCE}+\mathrm{FS}=16,4 \\
& 0 \mathrm{U}+0 \mathrm{CC}+0 \mathrm{FB}+0,8724 \mathrm{MM}+0,5808 \mathrm{SCE}+ \\
& 0,8164 \mathrm{FS}=11,89 \\
& 2,81 \mathrm{U}+0 \mathrm{CC}+0 \mathrm{FB}+0,0911 \mathrm{MM}+0,0584 \mathrm{SCE} \\
& +0,4878 \mathrm{FS}=2,486 \\
& 2,81 \mathrm{U}+0 \mathrm{CC}+0 \mathrm{FB}+0,0375 \mathrm{MM}+0,0292 \mathrm{SCE} \\
& +0,3188 \mathrm{FS}=1,583 \\
& 0 \mathrm{U}+0,3733 \mathrm{CC}+0,24 \mathrm{FB}+0,0003 \mathrm{MM}+ \\
& 0,0035 \mathrm{SCE}+0,0034 \mathrm{FS}=0,09625 \\
& 0 \mathrm{U}+0 \mathrm{CC}+0,1848 \mathrm{FB}+0,0025 \mathrm{MM}+0,0013 \\
& \quad \mathrm{SCE}+0,0058 \mathrm{FS}=0,06175
\end{aligned}
$$

Note que a equação 4 é referente a exigência de proteína degrada no rúmen. Transformando esse sistema de equações em matrizes, obtemos a seguinte matriz aumentada:

$$
\begin{array}{|cccccc:c|c}
1 & 1 & 1 & 1 & 1 & 1 & 16,4 \\
0 & 0 & 0 & 0,8724 & 0,5808 & 0,8164 & 11,89 \\
2,81 & 0 & 0 & 0,0911 & 0,0584 & 0,4878 & 2,486 \\
2,81 & 0 & 0 & 0,0375 & 0,0292 & 0,3188 & 1,583 \\
0 & 0,3733 & 0,24 & 0,0003 & 0,0035 & 0,0034 & 0,09625 \\
0 & 0 & 0,1848 & 0,0025 & 0,0013 & 0,0058 & 0,06175
\end{array}
$$

Observe que os elementos da diagonal principal nas colunas 2 e 3 são nulos. Assim vamos trocar a linha 2 pela linha 5 e trocar a linha 3 pela linha 6 . A matriz ficará assim:

\begin{tabular}{|cccccc:c}
\hdashline $1 ;$ & 1 & 1 & 1 & 1 & 1 & 16,4 \\
0 & 0,3733 & 0,24 & 0,0003 & 0,0035 & 0,0034 & 0,09625 \\
0 & 0 & 0,1848 & 0,0025 & 0,0013 & 0,0058 & 0,06175 \\
2,81 & 0 & 0 & 0,0375 & 0,0292 & 0,3188 & 1,583 \\
0 & 0 & 0 & 0,8724 & 0,5808 & 0,8164 & 11,89 \\
2,81 & 0 & 0 & 0,0911 & 0,0584 & 0,4878 & 2,486
\end{tabular} \mid

Agora vamos realizar as operações necessárias para escalonar essa matriz e possibilitar a 
resolução do sistema conforme exemplos anteriores.

$\mathrm{L} 4<=\mathrm{L} 4-2,81 \mathrm{~L} 1$
$\mathrm{~L} 6<=\mathrm{L} 6-2,81 \mathrm{~L} 1$

\begin{tabular}{|cccccc:c}
1 & 1 & 1 & 1 & 1 & 1 & 16,4 \\
0 & $-0,3733$ & 0,24 & 0,0003 & 0,0035 & 0,0034 & 0,09625 \\
0 & 0 & 0,1848 & 0,0025 & 0,0013 & 0,0058 & 0,06175 \\
0 & $-2,81$ & $-2,81$ & $-2,7725$ & $-2,7808$ & $-2,4912$ & $-44,501$ \\
0 & 0 & 0 & 0,8724 & 0,5808 & 0,8164 & 11,89 \\
0 & $-2,81$ & $-2,81$ & $-2,7189$ & $-2,7516$ & $-2,3222$ & $-43,598$
\end{tabular} \mid

$\mathrm{L} 4<=\mathrm{L} 4+7,5274 \mathrm{~L} 2$

$\mathrm{L} 6<=\mathrm{L} 6+7,5274 \mathrm{~L} 2$

\begin{tabular}{|cccccc:c}
1 & 1 & 1 & 1 & 1 & 1 & 16,4 \\
0 & 0,3733 & 0,24 & 0,0003 & 0,0035 & 0,0034 & 0,09625 \\
0 & 0 & 0,1848, & 0,0025 & 0,0013 & 0,0058 & 0,06175 \\
0 & 0 & $-1,0034$ & $-2,7702$ & $-2,7544$ & $-2,4656$ & $-43,776$ \\
0 & 0 & 0 & 0,8724 & 0,5808 & 0,8164 & 11,89 \\
0 & 0 & $-1,0034$ & $-2,7166$ & $-2,7252$ & $-2,2966$ & $-42,873$
\end{tabular} \mid

$\mathrm{L} 4<=\mathrm{L} 4+5,4296 \mathrm{~L} 3$

L6 <= L6 + 5,4296 L3

\begin{tabular}{|cccccc:c}
1 & 1 & 1 & 1 & 1 & 1 & 16,4 \\
0 & 0,3733 & 0,24 & 0,0003 & 0,0035 & 0,0034 & 0,09625 \\
0 & 0 & 0,1848 & 0,0025 & 0,0013 & 0,0058 & 0,06175 \\
0 & 0 & 0 & $(-2,7566 ;-2,7473$ & $-2,4341$ & $-43,4407$ \\
0 & 0 & 0 & 0,8724 & 0,5808 & 0,8164 & 11,89 \\
0 & 0 & 0 & $-2,703$ & $-2,7181$ & $-2,2651$ & $-42,5377$
\end{tabular} \mid

$\mathrm{L} 5<=3,1597 \mathrm{~L} 5+\mathrm{L} 4$

L6 $<=1,0198$ L6 - L4

\begin{tabular}{|cccccc:c}
1 & 1 & 1 & 1 & 1 & 1 & 16,4 \\
0 & 0,3733 & 0,24 & 0,0003 & 0,0035 & 0,0034 & 0,09625 \\
0 & 0 & 0,1848 & 0,0025 & 0,0013 & 0,0058 & 0,06175 \\
0 & 0 & 0 & $-2,7566$ & $-2,7473$ & $-2,4341$ & $-43,4407$ \\
0 & 0 & 0 & 0 & $-0,9121 ;$ & 0,1454 & $-5,8718$ \\
0 & 0 & 0 & 0 & $-0,0246$ & 0,1241 & 0,0607
\end{tabular} \mid

L6 $<=37,0772$ L6 - L5 \begin{tabular}{|cccccc:c}
1 & 1 & 1 & 1 & 1 & 1 & 16,4 \\
0 & 0,3733 & 0,24 & 0,0003 & 0,0035 & 0,0034 & 0,09625 \\
0 & 0 & 0,1848 & 0,0025 & 0,0013 & 0,0058 & 0,06175 \\
0 & 0 & 0 & $-2,7566$ & $-2,7473$ & $-2,4341$ & $-43,4407$ \\
0 & 0 & 0 & 0 & $-0,9121$ & 0,1454 & $-5,8718$ \\
0 & 0 & 0 & 0 & 0 & 4,4558 & 8,1223
\end{tabular}

Convertendo essa matriz escalonada em sistema, ficaria da seguinte forma:

$$
\begin{array}{r}
\mathrm{U}+\mathrm{CC}+\mathrm{FB}+\mathrm{MM}+\mathrm{SCE}+\mathrm{FS}=16,4 \\
0,3733 \mathrm{CC}+0,24 \mathrm{FB}+0,0003 \mathrm{MM}+0,0035 \mathrm{SCE} \\
+0,0034 \mathrm{FS}=0,09625 \\
0,1858 \mathrm{FB}+0,0025 \mathrm{MM}+0,0013 \mathrm{SCE}+0,0058 \\
\mathrm{FS}=0,06175 \\
-2,7566 \mathrm{MM}-2,7473 \mathrm{SCE}-2,4341 \mathrm{FS}=-43,4407 \\
-0,9121 \mathrm{SCE}+0,1454 \mathrm{FS}=-5,8718 \\
4,4558 \mathrm{FS}=8,1223
\end{array}
$$

Resolvendo o sistema:

$\mathrm{FS}=8,1223 / 4,4558$

$\mathrm{FS}=1,823 \mathrm{~kg}$ de $\mathrm{MS}$

$\mathrm{SCE}=\{-5,8718-(0,2650)\} /-0,9121$

$\mathrm{SCE}=6,728 \mathrm{~kg}$ de $\mathrm{MS}$

$\begin{aligned} \mathrm{MM}= & \{(-43,4407)+(2,4341 \times 1,823)+(2,7473 \\ & \text { × } 6,728)\} /-2,7566\end{aligned}$

$\mathrm{MM}=7,443 \mathrm{~kg}$ de $\mathrm{MS}$

$\mathrm{FB}=\{(0,06175)-(0,0058 \times 1,823)-(0,0013 \mathrm{x}$ $6,728)-(0,0025 \times 7,443)\} / 0,1858$

$\mathrm{FB}=0,128 \mathrm{~kg}$ de $\mathrm{MS}$

$\begin{aligned} \mathrm{CC}=\{ & (0,09625)-(0,0034 \times 1,823)-(0,0035 \times \\ & 6,728)-(0,0003 \times 7,443)-(0,24 \times 0,128) \\ & \} / 0,3733\end{aligned}$

$\mathrm{CC}=0,089 \mathrm{~kg}$ de $\mathrm{MS}$

$\mathrm{U}=16,4-1,823-6,728-7,443-0,128-0,089$

$\mathrm{U}=0,189 \mathrm{~kg}$ de $\mathrm{MS}$

Assim, obtemos a seguinte fórmula:

- Farelo de soja = 1,823 kg de MS

- Silagem de capim elefante $=6,728 \mathrm{~kg}$ de MS

- Milho moído = 7,443 kg de MS

- Fosfato bicálcico $=0,128 \mathrm{~kg}$ de MS 
- Calcário calcítico =0,089 kg de MS

- Ureia $=0,189 \mathrm{~kg}$ de MS

- Suplemento micromineral e vitamínico $=0,10 \mathrm{~kg}$

Total $=16,5 \mathrm{~kg}$ de MS

Verifica-se nessa fórmula uma relação volumoso:concentrado igual a 40:60, estando balanceada para energia, proteína bruta, proteína degradada no rúmen, cálcio, fósforo e demais microminerais e vitaminas suplementadas em 0,1 $\mathrm{kg}$ de margem deixada para essa finalidade, tudo isso calculado para uma capacidade de consumo equivalente a 16,5 $\mathrm{kg}$ de MS/dia. Na prática, essa margem de $0,1 \mathrm{~kg}$ seria ajustada de acordo com o suplemento disponível, e seu respectivo nível de inclusão, para ser utilizado na dieta. É válido ressaltar que a utilização de matrizes para formular rações ou dietas também possibilita estabelecer níveis de inclusão fixados de algum ingrediente, garantindo assim que o ingrediente esteja em um determinado nível na ração final.

Como exemplo, vamos refazer o cálculo anterior utilizando adicionalmente Silagem de milho (SM) fixado em $20 \%$ da dieta e Melaço de cana de açúcar (ME) fixado em 5\% para conferirem melhor digestibilidade e palatabilidade a dieta. Também vamos adicionar uma equação para balanceamento de sódio, utilizando $\mathrm{Sal}$ comum (SC) como principal fonte de sódio, mineral importante para a homeostase fisiológica animal. Vamos permanecer com a margem de 0,1 $\mathrm{kg}$ para inclusão de suplemento vitamínico e micromineral. Dessa forma, o sistema de equações ficaria assim:

$$
\begin{aligned}
& \mathrm{SC}+\mathrm{U}+\mathrm{CC}+\mathrm{FB}+\mathrm{MM}+\mathrm{SCE}+\mathrm{FS}+\mathrm{SM}+ \\
& \mathrm{ME}=16,4 \\
& 0 \mathrm{SC}+0 \mathrm{U}+0 \mathrm{CC}+0 \mathrm{FB}+0,8724 \mathrm{MM}+0,5808 \\
& \mathrm{SCE}+0,8164 \mathrm{FS}+0,6427 \mathrm{SM}+0,70 \mathrm{ME} \\
&=11,89 \\
& 0 \mathrm{SC}+2,81 \mathrm{U}+0 \mathrm{CC}+0 \mathrm{FB}+0,0911 \mathrm{MM}+ \\
& 0,0584 \mathrm{SCE}+0,4878 \mathrm{FS}+0,0726 \mathrm{SM}+ \\
& 0,0366 \mathrm{ME}=2,486 \\
& 0 \mathrm{SC}+ 2,81 \mathrm{U}+0 \mathrm{CC}+0 \mathrm{FB}+0,0375 \mathrm{MM}+ \\
& 0,0292 \mathrm{SCE}+0,3188 \mathrm{FS}+0,0535 \mathrm{SM}+ \\
& 0,0183 \mathrm{ME}=1,583 \\
& 0 \mathrm{SC}+ 0 \mathrm{U}+0,3733 \mathrm{CC}+0,24 \mathrm{FB}+0,0003 \mathrm{MM} \\
&+ 0,0035 \mathrm{SCE}+0,0034 \mathrm{FS}+0,003 \mathrm{SM}+ \\
& 0 \mathrm{ME}=0,09625
\end{aligned}
$$

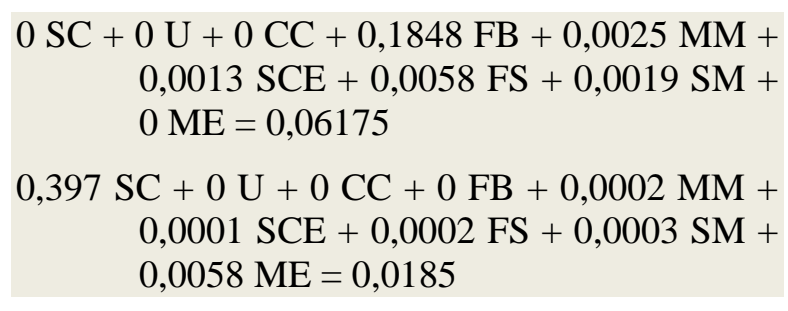

Observe a grande complexidade que o sistema apresenta para ser revolvido por meio não matricial. A matriz aumentada desse sistema é:

\begin{tabular}{|ccccccccc:c}
1 & 1 & 1 & 1 & 1 & 1 & 1 & 1 & 1 & 16,4 \\
0 & 0 & 0 & 0 & 0,8724 & 0,5808 & 0,8164 & 0,6427 & 0,7 & 11,89 \\
0 & 2,81 & 0 & 0 & 0,0911 & 0,0584 & 0,4878 & 0,0726 & 0,0366 & 2,486 \\
0 & 2,81 & 0 & 0 & 0,0375 & 0,0292 & 0,3188 & 0,0535 & 0,0183 & 1,583 \\
0 & 0 & 0,3733 & 0,24 & 0,0003 & 0,00350 & 0,0034 & 0,003 & 0 & 0,09625 \\
0 & 0 & 0 & 0,1848 & 0,0025 & 0,0013 & 0,0058 & 0,0019 & 0 & 0,06175 \\
0,397 & 0 & 0 & 0 & 0,0002 & 0,0001 & 0,0002 & 0,0003 & 0,0058 & 0,0185
\end{tabular} \mid

Perceba que essa matriz possui uma ordem $7 \mathrm{X}$ 9 (sete linhas e nove colunas), não sendo, portanto, uma matriz quadrada. Contudo, lembre-se que os alimentos silagem de milho (SM) e melaço de cana de açúcar (ME) foram fixados em $20 \%$ e 5 $\%$, respectivamente. Assim, vamos voltar ao sistema de equações lineares e calcular a participação (em kg) de cada alimento. Após isso, as constantes geradas pelos alimentos silagem de milho e melaço de cana de açúcar devem ser descontadas dos termos independentes de todas as equações. Inicialmente, vamos calcular as constantes geradas nas equações pela SM e ME. Seguem os cálculos:

$$
\begin{aligned}
& \mathrm{SC}+\mathrm{U}+\mathrm{CC}+\mathrm{FB}+\mathrm{MM}+\mathrm{SCE}+\mathrm{FS}+3,28+ \\
& 0,82=16,4 \\
& 0 \mathrm{SC}+0 \mathrm{U}+0 \mathrm{CC}+0 \mathrm{FB}+0,8724 \mathrm{MM}+0,5808 \\
& \mathrm{SCE}+0,8164 \mathrm{FS}+(0,6427 \times 3,28)+ \\
&(0,70 \times 0,82)=11,89 \\
& 0 \mathrm{SC}+2,81 \mathrm{U}+0 \mathrm{CC}+0 \mathrm{FB}+0,0911 \mathrm{MM}+ \\
& 0,0584 \mathrm{SCE}+0,4878 \mathrm{FS}+(0,0726 \times 3,28) \\
&+(0,0366 \times 0,82)=2,486 \\
& 0 \mathrm{SC}+ 2,81 \mathrm{U}+0 \mathrm{CC}+0 \mathrm{FB}+0,0375 \mathrm{MM}+ \\
& 0,0292 \mathrm{SCE}+0,3188 \mathrm{FS}+(0,0535 \times 3,28) \\
&+(0,0183 \times 0,82)=1,583 \\
& 0 \mathrm{SC}+ 0 \mathrm{U}+0,3733 \mathrm{CC}+0,24 \mathrm{FB}+0,0003 \mathrm{MM} \\
&+0,0035 \mathrm{SCE}+0,0034 \mathrm{FS}+(0,003 \times \\
&3,28)+(0 \times 0,82)=0,09625 \\
& 0 \mathrm{SC}+ 0 \mathrm{U}+0 \mathrm{CC}+0,1848 \mathrm{FB}+0,0025 \mathrm{MM}+ \\
& 0,0013 \mathrm{SCE}+0,0058 \mathrm{FS}+(0,0019 \times 3,28) \\
&+(0 \times 0,82)=0,06175
\end{aligned}
$$




$$
\begin{array}{r}
0,397 \mathrm{SC}+0 \mathrm{U}+0 \mathrm{CC}+0 \mathrm{FB}+0,0002 \mathrm{MM}+ \\
0,0001 \mathrm{SCE}+0,0002 \mathrm{FS}+(0,0003 \times 3,28) \\
+(0,0058 \times 0,82)=0,0185
\end{array}
$$

Observe que os $20 \%$ fixados de silagem de milho são equivalentes ao valor $3,28(0,2 \times 16,4)$ que substituiu a incógnita SM nas equações. Da mesma forma, os $5 \%$ fixados de melaço são equivalentes ao valor $0,82(0,05 \times 16,4)$ que também substituiu a incógnita ME. Agora, vamos descontar, dos termos independentes, os produtos da multiplicação dos coeficientes pelos valores de SM e ME. Seria da seguinte forma:

$$
\begin{aligned}
& \mathrm{SC}+\mathrm{U}+\mathrm{CC}+\mathrm{FB}+\mathrm{MM}+\mathrm{SCE}+\mathrm{FS}=16,4- 3,28-0,82 \\
& 0 \mathrm{SC}+ 0 \mathrm{U}+0 \mathrm{CC}+0 \mathrm{FB}+0,8724 \mathrm{MM}+0,5808 \\
& \mathrm{SCE}+0,8164 \mathrm{FS}=11,89-(0,6427 \times \\
&3,28)-(0,70 \times 0,82) \\
& 0 \mathrm{SC}+ 2,81 \mathrm{U}+0 \mathrm{CC}+0 \mathrm{FB}+0,0911 \mathrm{MM}+ \\
& 0,0584 \mathrm{SCE}+0,4878 \mathrm{FS}=2,486- \\
&(0,0726 \times 3,28)-(0,0366 \times 0,82) \\
& 0 \mathrm{SC}+ 2,81 \mathrm{U}+0 \mathrm{CC}+0 \mathrm{FB}+0,0375 \mathrm{MM}+ \\
& 0,0292 \mathrm{SCE}+0,3188 \mathrm{FS}=1,583-(0,0535 \\
&\mathrm{x} 3,28)-(0,0183 \times 0,82) \\
& 0 \mathrm{SC}+ 0 \mathrm{U}+0,3733 \mathrm{CC}+0,24 \mathrm{FB}+0,0003 \mathrm{MM} \\
&+0,0035 \mathrm{SCE}+0,0034 \mathrm{FS}=0,09625- \\
&(0,003 \times 3,28)-(0 \times 0,82) \\
& 0 \mathrm{SC}+ 0 \mathrm{U}+0 \mathrm{CC}+0,1848 \mathrm{FB}+0,0025 \mathrm{MM}+ \\
& 0,0013 \mathrm{SCE}+0,0058 \mathrm{FS}=0,06175- \\
&(0,0019 \times 3,28)-(0 \times 0,82) \\
& 0,397 \mathrm{SC}+0 \mathrm{U}+0 \mathrm{CC}+0 \mathrm{FB}+0,0002 \mathrm{MM}+ \\
& 0,0001 \mathrm{SCE}+0,0002 \mathrm{FS}=0,0185- \\
&(0,0003 \times 3,28)-(0,0058 \times 0,82)
\end{aligned}
$$

\begin{tabular}{|c|c|c|c|c|c|c|c|}
\hline 1 & 1 & 1 & 1 & 1 & 1 & 1 & 12,3000 \\
\hline 0 & 0 & 0 & 0 & 0,8724 & 0,5808 & 0,8164 & 9,2079 \\
\hline 0 & 2,81 & 0 & 0 & 0,0911 & 0,0584 & 0,4878 & 2,2179 \\
\hline 0 & 2,81 & 0 & 0 & 0,0375 & 0,0292 & 0,3188 & 1,3925 \\
\hline 0 & 0 & 0,3733 & 0,24 & 0,0003 & 0,00350 & 0,0034 & 0,0864 \\
\hline 0 & 0 & 0 & 0,1848 & 0,0025 & 0,0013 & 0,0058 & 0,0555 \\
\hline 0,397 & 0 & 0 & 0 & 0,0002 & 0,0001 & 0,0002 & 0,0128 \\
\hline
\end{tabular}

\begin{tabular}{|c|c|c|c|c|c|c|c|}
\hline 1 & 1 & 1 & 1 & 1 & 1 & 1 & 12,3000 \\
\hline 0 & 0 & 0 & 0 & 0,8724 & 0,5808 & 0,8164 & 9,2079 \\
\hline 0 & 2,81 & 0 & 0 & 0,0911 & 0,0584 & 0,4878 & 2,2179 \\
\hline 0 & 2,81 & 0 & 0 & 0,0375 & 0,0292 & 0,3188 & 1,3925 \\
\hline 0 & 0 & 0,3733 & 0,24 & 0,0003 & 0,00350 & 0,0034 & 0,0864 \\
\hline 0 & 0 & 0 & 0,1848 & 0,0025 & 0,0013 & 0,0058 & 0,0555 \\
\hline 0 & -1 & -1 & -1 & $-0,9994$ & $-0,9997$ & $-0,9994$ & $-12,268$ \\
\hline
\end{tabular}

\begin{tabular}{|c|c|c|c|c|c|c|c|}
\hline 1 & 1 & 1 & 1 & 1 & 1 & 1 & 12,3000 \\
\hline 0 & 2,81 & 0 & 0 & 0,0911 & 0,0584 & 0,4878 & 2,2179 \\
\hline 0 & 0 & 0 & 0 & 0,8724 & 0,5808 & 0,8164 & 9,2079 \\
\hline 0 & 0 & 0 & 0 & $-0,0536$ & $-0,0292$ & $-0,169$ & $-0,8254$ \\
\hline 0 & 0 & 0,3733 & 0,24 & 0,0003 & 0,00350 & 0,0034 & 0,0864 \\
\hline 0 & 0 & 0 & 0,1848 & 0,0025 & 0,0013 & 0,0058 & 0,0555 \\
\hline 0 & -1 & -1 & -1 & $-0,9994$ & $-0,9997$ & $-0,9994$ & $-12,268$ \\
\hline
\end{tabular}

Resolvendo parcialmente as equações:

$$
\begin{aligned}
& \mathrm{SC}+\mathrm{U}+\mathrm{CC}+\mathrm{FB}+\mathrm{MM}+\mathrm{SCE}+\mathrm{FS}=12,3 \\
& 0 \mathrm{SC}+0 \mathrm{U}+0 \mathrm{CC}+0 \mathrm{FB}+0,8724 \mathrm{MM}+0,5808 \\
& \mathrm{SCE}+0,8164 \mathrm{FS}=9,2079 \\
& 0 \mathrm{SC}+ 2,81 \mathrm{U}+0 \mathrm{CC}+0 \mathrm{FB}+0,0911 \mathrm{MM}+ \\
& 0,0584 \mathrm{SCE}+0,4878 \mathrm{FS}=2,2179 \\
& 0 \mathrm{SC}+ 2,81 \mathrm{U}+0 \mathrm{CC}+0 \mathrm{FB}+0,0375 \mathrm{MM}+ \\
& 0,0292 \mathrm{SCE}+0,3188 \mathrm{FS}=1,3925 \\
& 0 \mathrm{SC}+0 \mathrm{U}+0,3733 \mathrm{CC}+0,24 \mathrm{FB}+0,0003 \mathrm{MM} \\
&+ 0,0035 \mathrm{SCE}+0,0034 \mathrm{FS}=0,0864 \\
& 0 \mathrm{SC}+ 0 \mathrm{U}+0 \mathrm{CC}+0,1848 \mathrm{FB}+0,0025 \mathrm{MM}+ \\
& 0,0013 \mathrm{SCE}+0,0058 \mathrm{FS}=0,0555
\end{aligned}
$$

\section{$0,397 \mathrm{SC}+0 \mathrm{U}+0 \mathrm{CC}+0 \mathrm{FB}+0,0002 \mathrm{MM}+$ $0,0001 \mathrm{SCE}+0,0002 \mathrm{FS}=0,0128$}

Note que as quantidades de silagem de milho e melaço de cana de açúcar fixados já possibilitou descontar a participação desses alimentos nas equações da matéria seca e demais nutrientes do sistema. Agora, o sistema deverá ser solucionado para as outras incógnitas. A matriz aumentada desse novo sistema é:

Note que essa nova matriz possui ordem $7 \times 7$, sendo portanto uma matriz quadrada, podendo ser escalonada pelo método da eliminação de Gauss demonstrado anteriormente. Vamos agora aplicar as operações matemáticas necessárias para escalonar essa matriz:

$$
\text { L7 }<=2,5188 \mathrm{~L} 7-\mathrm{L} 1
$$

$$
\begin{aligned}
& \text { L2 } \Leftrightarrow \text { L3 } \\
& \text { L4 }<=\text { L4 }- \text { L2 }
\end{aligned}
$$




$$
\begin{aligned}
& \text { L2 } \Leftrightarrow \text { L7 } \\
& \text { L3 } \Leftrightarrow \text { L5 } \\
& \text { L7 }<=0,3558 \text { L7 + L2 }
\end{aligned}
$$$$
\begin{array}{|ccccccc:c}
1 & 1 & 1 & 1 & 1 & 1 & 1 & 12,3000 \\
0 & -1 & -1 & -1 & -0,9994 & -0,9997 & -0,9994 & -12,2677 \\
0 & 0 & 0,3733 & 0,24 & 0,0003 & 0,0035 & 0,0034 & 0,0864 \\
0 & 0 & 0 & 0 & -0,0536 & -0,0292 & -0,169 & -0,8254 \\
0 & 0 & 0 & 0 & 0,8724 & 0,5808 & 0,8164 & 9,2079 \\
0 & 0 & 0 & 0,1848 & 0,0025 & 0,0013 & 0,0058 & 0,0555 \\
0 & 0 & -1 & -1 & -0,9669 & -0,9786 & -0,8258 & -11,4788
\end{array}
$$$$
\mathrm{L} 7<=0,3733 \mathrm{~L} 7+\mathrm{L} 3
$$

\section{L4 $\Leftrightarrow$ L6}

$$
\begin{array}{|ccccccc:c}
1 & 1 & 1 & 1 & 1 & 1 & 1 & 12,3000 \\
0 & -1 & -1 & -1 & -0,9994 & -0,9997 & -0,9994 & -12,2677 \\
0 & 0 & 0,3733 & 0,24 & 0,0003 & 0,0035 & 0,0034 & 0,0864 \\
0 & 0 & 0 & 0,1848 & 0,0025 & 0,0013 & 0,0058 & 0,0555 \\
0 & 0 & 0 & 0 & 0,8724 & 0,5808 & 0,8164 & 9,2079 \\
0 & 0 & 0 & 0 & -0,0536 & -0,0292 & -0,169 & -0,8254 \\
0 & 0 & 0 & -0,1333 & -0,3606 & -0,3618 & -0,3048 & -4,1986
\end{array} \mid
$$

\section{$\mathrm{L} 7<=1,3863 \mathrm{~L} 7+\mathrm{L} 4$}

\begin{tabular}{|ccccccc:c}
1 & 1 & 1 & 1 & 1 & 1 & 1 & 12,3000 \\
0 & -1 & -1 & -1 & $-0,9994$ & $-0,9997$ & $-0,9994$ & $-12,2677$ \\
0 & 0 & 0,3733 & 0,24 & 0,0003 & 0,0035 & 0,0034 & 0,0864 \\
0 & 0 & 0 & 0,1848 & 0,0025 & 0,0013 & 0,0058 & 0,0555 \\
0 & 0 & 0 & 0 & 0,8724 & 0,5808 & 0,8164 & 9,2079 \\
0 & 0 & 0 & 0 & $-0,0536$ & $-0,0292$ & $-0,169$ & $-0,8254$ \\
0 & 0 & 0 & 0 & $-0,4973$ & $-0,4966$ & $-0,4167$ & $-5,765$
\end{tabular} \mid

\section{L6 $<=16,2761 \mathrm{~L} 6+\mathrm{L} 5$}

L7 $<=1,7542 \mathrm{~L} 7+\mathrm{L} 5$

\begin{tabular}{|ccccccc:c}
1 & 1 & 1 & 1 & 1 & 1 & 1 & 12,3000 \\
0 & -1 & -1 & -1 & $-0,9994$ & $-0,9997$ & $-0,9994$ & $-12,2677$ \\
0 & 0 & 0,3733 & 0,24 & 0,0003 & 0,0035 & 0,0034 & 0,0864 \\
0 & 0 & 0 & 0,1848 & 0,0025 & 0,0013 & 0,0058 & 0,0555 \\
0 & 0 & 0 & 0 & 0,8724 & 0,5808 & 0,8164 & 9,2079 \\
0 & 0 & 0 & 0 & 0 & 0,1055 & $-1,9342$ & $-4,2263$ \\
0 & 0 & 0 & 0 & 0 & $-0,2903$ & 0,0854 & $-0,9050$
\end{tabular} \mid

$\mathrm{L} 7<=0,3634 \mathrm{~L} 7+\mathrm{L} 6$

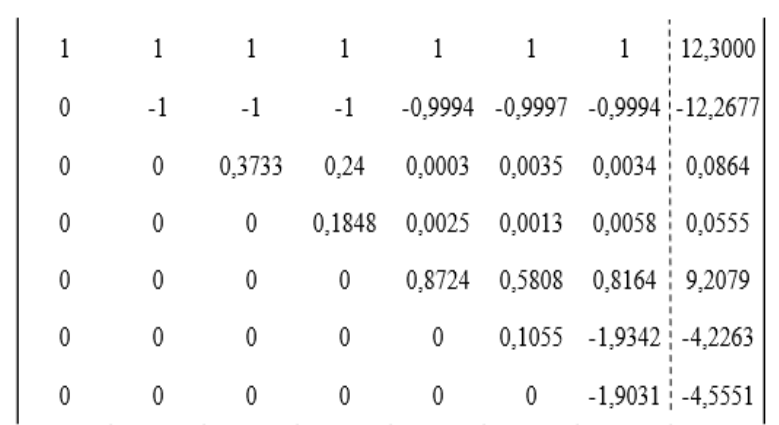

Após a matriz escalonada, vamos convertê-la novamente em sistema de equações:

$$
\begin{array}{r}
\mathrm{SC}+\mathrm{U}+\mathrm{CC}+\mathrm{FB}+\mathrm{MM}+\mathrm{SCE}+\mathrm{FS}=12,3 \\
-\mathrm{U}-\mathrm{CC}-\mathrm{FB}-0,9994 \mathrm{MM}-0,9997 \mathrm{SCE}- \\
0,9994 \mathrm{FS}=-12,2677 \\
0,3733 \mathrm{CC}+0,24 \mathrm{FB}+0,0003 \mathrm{MM}+0,0035 \mathrm{SCE} \\
+0,0034 \mathrm{FS}=0,0864 \\
0,1848 \mathrm{FB}+0,0025 \mathrm{MM}+0,0013 \mathrm{SCE}+0,0058 \\
\mathrm{FS}=0,0555 \\
0,8724 \mathrm{MM}+0,5808 \mathrm{SCE}+0,8164 \mathrm{FS}=9,2079 \\
0,1055 \mathrm{SCE}-1,9342 \mathrm{FS}=-4,2263 \\
-1,9031 \mathrm{FS}=-4,5551
\end{array}
$$

Resolvendo o sistema:

$\mathrm{FS}=(-4,5551) /(-1,9031)$

$\mathrm{FS}=2,393 \mathrm{~kg}$ de MS

$\mathrm{SCE}=\{(-4,2263)+(1,9342 \times 2,393)\} / 0,1055$

$\mathrm{SCE}=3,812 \mathrm{~kg}$ de MS

$\mathrm{MM}=\{9,2079-(0,8164 \times 2,393)-(0,5808 \times$ $3,812)\} / 0,8724$

$\mathrm{MM}=5,777 \mathrm{~kg}$ de MS

$\mathrm{FB}=\{0,0555-(0,0058 \times 2,393)-(0,0013 \times$ $3,812)-(0,0025 \times 5,777)\} / 0,1848$

$\mathrm{FB}=0,120 \mathrm{~kg}$ de $\mathrm{MS}$

$$
\begin{aligned}
& \mathrm{CC}=\{0,0864-(0,0034 \times 2,393)-(0,0035 \times \\
& 3,812)-(0,0003 \times 5,777)-(0,24 \times \\
& 0,120)\} / 0,3733 \\
& \text { - } U=\{-12,2677+(0,9994 \times 2,393)+(0,9997 \times \\
& 3,812)+(0,9994 \times 5,777)+(0,120)+ \\
& (0,092)\}
\end{aligned}
$$

$\mathrm{U}=0,079 \mathrm{~kg}$ de $\mathrm{MS}$

$\mathrm{SC}=12,3-2,393-3,812-5,777-0,120-0,092$ $-0,079$ 


\section{$\mathrm{SC}=0,027 \mathrm{~kg}$ de MS}

Após a solução das incógnitas, obtemos a seguinte fórmula:

- Silagem de milho $=3,28 \mathrm{~kg}$ de MS

- Melaço de cana de açúcar =0,82 kg de MS

- Farelo de soja = 2,393 kg de MS

- Silagem de capim elefante $=3,812 \mathrm{~kg}$ de MS

- Milho moído = 5,777 kg de MS

- Fosfato bicálcico $=0,120 \mathrm{~kg}$ de MS

- Calcário calcítico =0,092 kg de MS

- Ureia $=0,079 \mathrm{~kg}$ de MS

- Sal comum $=0,027 \mathrm{~kg}$ de MS

- Suplemento micromineral e vitamínico $=0,1 \mathrm{~kg}$

Total $=16,5 \mathrm{~kg}$ de MS

Para converter os valores de termos quantitativos $(\mathrm{kg})$ para termos percentuais (\%) é necessário apenas dividir a quantidade calculada de cada alimento pelo consumo de matéria seca $(16,5)$ e multiplicar por 100. Ressalta-se que, quando verificado se os níveis nutricionais (NDT, PB, PDR, Ca, P e Na) estão atendidos de acordo com a exigência, pode haver pequenas diferenças a partir da segunda casa decimal devido a não utilização das frações numéricas completas durante os cálculos, o que tornaria as operações matemáticas exaustivas caso fossem utilizadas. Contudo, essa diferença é pequena e irrelevante.

\section{Método do Escalonamento de Gauss-Jordan}

Perceba que, após a obtenção da matriz escalonada pelo método da eliminação de Gauss, convertemos novamente a matriz escalonada em sistema para então resolvermos as equações. No entanto, poderíamos ir mais longe no escalonamento da matriz. Com o método da eliminação de Gauss obtemos a matriz escalonada de forma que todos os números a baixo da diagonal principal sejam nulos. Com tudo, existe um método chamado escalonamento de GaussJordan que, além de zerar todos os valores abaixo da diagonal principal, também tornam nulos todos os valores a cima da diagonal principal. Ao final do escalonamento de Gauss-Jordan iremos obter uma matriz com valores não nulos apenas na diagonal principal, sendo cada coluna correspondente a uma incógnita do sistema de equações que deu origem a matriz. Esse método irá produzir uma matriz que, quando convertida em sistema novamente, terá uma resolução mais simplificada.

Para o exemplo anterior, utilizar o escalonamento de Gauss-Jordan é necessário apenas continuar o escalonamento da matriz de forma a zerar os valores que estão a cima da diagonal principal, haja vista que os valores a baixo da diagonal principal já estão zerados. A matriz que vamos continuar escalonando e as operações necessárias para zerar os valores a cima da diagonal principal são:

\begin{tabular}{|ccccccc:c}
1 & 1 & 1 & 1 & 1 & 1 & 1 & 12,3 \\
0 & -1 & -1 & -1 & $-0,9994$ & $-0,9997$ & $-0,9994$ & $-12,2677$ \\
0 & 0 & 0,3733 & 0,24 & 0,0003 & 0,0035 & 0,0034 & 0,0864 \\
0 & 0 & 0 & 0,1848 & 0,0025 & 0,0013 & 0,0058 & 0,0555 \\
0 & 0 & 0 & 0 & 0,8724 & 0,5808 & 0,8164 & 9,2079 \\
0 & 0 & 0 & 0 & 0 & 0,1055 & $-1,9342$ & $-4,2263$ \\
0 & 0 & 0 & 0 & 0 & 0 & $-1,9031$ & $-4,5551$
\end{tabular}

$\mathrm{L} 1<=\mathrm{L} 1+\mathrm{L} 2$

$\mathrm{L} 2<=0,3733 \mathrm{~L} 2+\mathrm{L} 3$

\begin{tabular}{|ccccccc:c}
1 & 0 & 0 & 0 & 0,0006 & 0,0003 & 0,0006 & 0,0323 \\
0 & $-0,3733$ & 0 & $-0,1333$ & $-0,3728$ & $-0,3697$ & $-0,3697$ & $-4,4931$ \\
0 & 0 & 0,3733 & 0,24 & 0,0003 & 0,0035 & 0,0034 & 0,0864 \\
0 & 0 & 0 & 0,1848 & 0,0025 & 0,0013 & 0,0058 & 0,0555 \\
0 & 0 & 0 & 0 & 0,8724 & 0,5808 & 0,8164 & 9,2079 \\
0 & 0 & 0 & 0 & 0 & 0,1055 & $-1,9342$ & $-4,2263$ \\
0 & 0 & 0 & 0 & 0 & 0 & $-1,9031$ & $-4,5551$
\end{tabular}

$\mathrm{L} 2<=1,3863 \mathrm{~L} 2+\mathrm{L} 4$

L3 $<=0,77$ L3 - L4

\begin{tabular}{|ccccccc:c}
1 & 0 & 0 & 0 & 0,0006 & 0,0003 & 0,0006 & 0,0323 \\
0 & $-0,5175$ & 0 & 0 & $-0,5143$ & $-0,5112$ & $-0,5067$ & $-6,1733$ \\
0 & 0 & 0,2874 & 0 & $-0,0023$ & 0,0014 & $-0,0032$ & 0,0110 \\
0 & 0 & 0 & 0,1848 & 0,0025 & 0,0013 & 0,0058 & 0,0555 \\
0 & 0 & 0 & 0 & 0,8724 & 0,5808 & 0,8164 & 9,2079 \\
0 & 0 & 0 & 0 & 0 & 0,1055 & $-1,9342$ & $-4,2263$ \\
0 & 0 & 0 & 0 & 0 & 0 & $-1,9031$ & $-4,5551$
\end{tabular} \mid

$\mathrm{L} 1<=1454 \mathrm{~L} 1-\mathrm{L} 5$

$\mathrm{L} 2<=1,6962 \mathrm{~L} 2+\mathrm{L} 5$ 


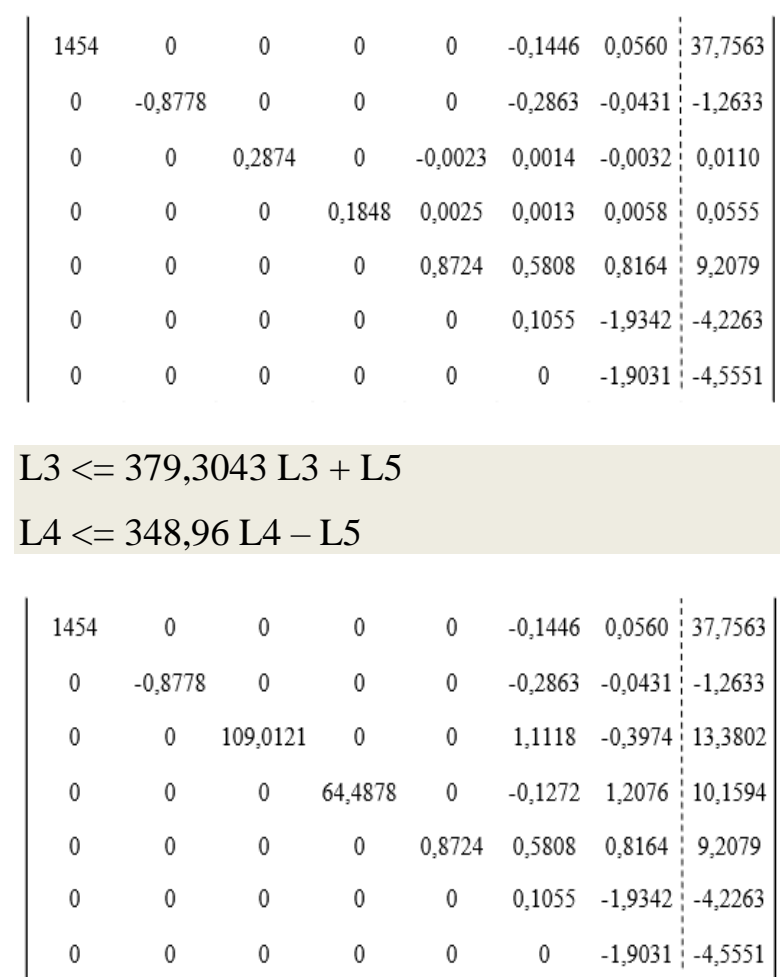

$\mathrm{L} 1<=0,7295 \mathrm{~L} 1+\mathrm{L} 6$

$\mathrm{L} 2<=0,3684 \mathrm{~L} 2+\mathrm{L} 6$

L3 $<=0,0948$ L3 - L6

\begin{tabular}{|ccccccc:c}
1060,693 & 0 & 0 & 0 & 0 & 0 & $-1,8933$ & 23,3169 \\
0 & $-0,3234$ & 0 & 0 & 0 & 0 & $-1,9501$ & $-4,6917$ \\
0 & 0 & 10,3343 & 0 & 0 & 0 & 1,8965 & 5,4947 \\
0 & 0 & 0 & 64,4878 & 0 & $-0,1272$ & 1,2076 & 10,1594 \\
0 & 0 & 0 & 0 & 0,8724 & 0,5808 & 0,8164 & 9,2079 \\
0 & 0 & 0 & 0 & 0 & 0,1055 & $-1,9342$ & $-4,2263$ \\
0 & 0 & 0 & 0 & 0 & 0 & $-1,9031$ & $-4,5551$
\end{tabular} \mid

L4 $<=0,8294 \mathrm{~L} 4+\mathrm{L} 6$

L5 $<=0,1816$ L5 - L6

\begin{tabular}{|ccccccc:c}
1060,693 & 0 & 0 & 0 & 0 & 0 & $-1,8933$ & 23,3169 \\
0 & $-0,3234$ & 0 & 0 & 0 & 0 & $-1,9501$ & $-4,6917$ \\
0 & 0 & 10,3343 & 0 & 0 & 0 & 1,8965 & 5,4947 \\
0 & 0 & 0 & 53,4862 & 0 & 0 & $-0,9326$ & 4,1999 \\
0 & 0 & 0 & 0 & 0,1584 & 0 & 2,0825 & 5,8985 \\
0 & 0 & 0 & 0 & 0 & 0,1055 & $-1,9342$ & $-4,2263$ \\
0 & 0 & 0 & 0 & 0 & 0 & $-1,9031$ & $-4,5551$
\end{tabular} \mid

$\mathrm{L} 1<=1,0051 \mathrm{~L} 1-\mathrm{L} 7$

$\mathrm{L} 2<=0,9758 \mathrm{~L} 2-\mathrm{L} 7$

$\mathrm{L} 3<=1,0034 \mathrm{~L} 3+\mathrm{L} 7$ \begin{tabular}{|ccccccc:c}
1066,103 & 0 & 0 & 0 & 0 & 0 & 0 & 27,9909 \\
0 & $-0,3156$ & 0 & 0 & 0 & 0 & 0 & $-0,0231$ \\
0 & 0 & 10,3694 & 0 & 0 & 0 & 0 & 0,9583 \\
0 & 0 & 0 & 53,4862 & 0 & 0 & $-0,9326$ & 4,1999 \\
0 & 0 & 0 & 0 & 0,1584 & 0 & 2,0825 & 5,8985 \\
0 & 0 & 0 & 0 & 0 & 0,1055 & $-1,9342$ & $-4,2263$ \\
0 & 0 & 0 & 0 & 0 & 0 & $-1,9031$ & $-4,5551$
\end{tabular} \mid

\section{L4 $<=2,0406$ L4 - L7 \\ $\mathrm{L} 5<=0,9138 \mathrm{~L} 5+\mathrm{L} 7$ \\ L6 $<=0,9839$ L6 - L7}

\begin{tabular}{|ccccccc:c}
1066,103 & 0 & 0 & 0 & 0 & 0 & 0 & 27,9909 \\
0 & $-0,3156$ & 0 & 0 & 0 & 0 & 0 & $-0,0231$ \\
0 & 0 & 10,3694 & 0 & 0 & 0 & 0 & 0,9583 \\
0 & 0 & 0 & 109,1439 & 0 & 0 & 0 & 13,1254 \\
0 & 0 & 0 & 0 & 0,1447 & 0 & 0 & 0,8349 \\
0 & 0 & 0 & 0 & 0 & 0,1038 & 0 & 0,3968 \\
0 & 0 & 0 & 0 & 0 & 0 & $-1,9031$ & $-4,5551$
\end{tabular} \mid

Após a matriz escalonada, perceba que todos os valores a baixo e a cima da diagonal principal estão zerados. Note também que cada valor da diagonal principal corresponde ao coeficiente de uma incógnita e possuindo um valor dos termos independentes correspondente. Assim, a solução de cada incógnita é encontrada dividindo-se o valor do termo independente referente aquela incógnita pelo seu coeficiente. Convertendo essa matriz em sistema ficaria da seguinte forma:

$-1,9031 \mathrm{FS}=-4,5551$

0,1038 SCE $=0,3968$

$0,1447 \mathrm{MM}=0,8349$

$109,1439 \mathrm{FB}=13,1254$

$10,3694 \mathrm{CC}=0,9583$

$-0,3156 \mathrm{U}=-0,0231$

$1066,103 \mathrm{SC}=27,9909$

Resolvendo o sistema:

$\mathrm{FS}=2,393 \mathrm{~kg}$ de MS

$\mathrm{SCE}=3,823 \mathrm{~kg}$ de MS

$\mathrm{MM}=5,769 \mathrm{~kg}$ de $\mathrm{MS}$

$\mathrm{FB}=0,120 \mathrm{~kg}$ de MS

$\mathrm{CC}=0,092 \mathrm{~kg}$ de $\mathrm{MS}$ 
$\mathrm{U}=0,073 \mathrm{~kg}$ de $\mathrm{MS}$

$\mathrm{SC}=0,026 \mathrm{~kg}$ de $\mathrm{MS}$

A esse resultado, deve-se incluir os valores referentes a silagem de milho, melaço de cana de açúcar e suplemento micromineral e vitamínico pré-fixados para se ter a fórmula completa. Verifique que ao utilizar o escalonamento de Gauss-Jordan as incógnitas obtiveram os mesmos valores encontrados pelo método da eliminação de Gauss utilizado anteriormente. Apenas algumas incógnitas tiveram uma pequena e irrelevante diferença numérica entre os diferentes métodos, a partir da segunda casa decimal, devido a não utilização das frações numéricas completas como já comentado anteriormente. Dessa forma, o escalonamento de Gauss-Jordan também possibilita a resolução de sistemas de equações lineares complexos utilizados em formulação de rações.

\section{Considerações Finais}

As operações matriciais possibilitam a resolução de sistemas lineares complexos utilizados para formular rações, sendo mais uma possibilidade matemática que os técnicos nutricionistas podem utilizar cotidianamente. Com essa metodologia, não há limites de parâmetros e incógnitas utilizados para formular rações mais completas, possibilitando o completo atendimento das exigências nutricionais requeridas.

\section{Referências Bibliográficas}

Lana, R. P. 2007. Sistema Viçosa de formulação de rações. Universidade Federal de Viçosa, Viçosa, Minas Gerais.

NRC. 1989. Nutrient Requirements of Dairy Cattle, 7th rev. edn. Natl. Acad. Press, Washington, DC., Washington.

NRC. 2001. Nutrient Requirements of Dairy Cattle, 7th rev. edn. Natl. Acad. Press, Washington, DC., Washington.

Rostagno, H. S., Albino, L. F. T., Donzele, J. L., Gomes, P. C., Oliveira, R., Lopes, D. C., Ferreira, A. S., Barreto, S. \& Euclides, R. F. 2011. Composição de alimentos e exigências nutricionais, 3 edn. Universidade Federal de Viçosa, Viçosa.

Valadares Filho, S. C. 2006. Tabelas brasileiras de composição de alimentos para bovinos. UFV, Viçosa.

Article History:

Received 17 May 2017

Accepted 29 June 2017

Available online 29 August 2017

License information: This is an open-access article distributed under the terms of the Creative Commons Attribution License 4.0, which permits unrestricted use, distribution, and reproduction in any medium, provided the original work is properly cited. 